Spatial cognitive implications of teleporting through virtual environments

Lucia A. Cherep, Alex F. Lim, Jonathan W. Kelly, Devi Acharya, Alfredo Velasco, Emanuel Bustamante, Alec G. Ostrander, Stephen B. Gilbert Iowa State University

Accepted for publication in Journal of Experimental Psychology: Applied http://dx.doi.org/10.1037/xap0000263

Author Note

Pre-registrations for Experiments $4 \& 5$, as well as videos, data, supplementary analyses, and experiment code are available on the Open Science Framework (https://osf.io/m4zfv/). This material is based upon work supported by the National Science Foundation under Grant No. CHS-1816029.

Correspondence concerning this article should be addressed to Jonathan Kelly, Department of Psychology, Iowa State University, Ames, IA, 50011-1041. Contact: jonkelly@iastate.edu. 


\begin{abstract}
Teleporting is a popular interface to allow virtual reality users to explore environments that are larger than the available walking space. When teleporting, the user positions a marker in the virtual environment and is instantly transported without any self-motion cues. Five experiments were designed to evaluate the spatial cognitive consequences of teleporting and to identify environmental cues that could mitigate those costs. Participants performed a triangle completion task by traversing two outbound path legs before pointing to the unmarked path origin. Locomotion was accomplished via walking or two common implementations of the teleporting interface distinguished by the concordance between movement of the body and movement through the virtual environment. In the partially concordant teleporting interface, participants teleported to translate (change position) but turned the body to rotate. In the discordant teleporting interface, participants teleported to translate and rotate. Across all five experiments, discordant teleporting produced larger errors than partially concordant teleporting which produced larger errors than walking, reflecting the importance of translational and rotational selfmotion cues. Furthermore, geometric boundaries (room walls or a fence) were necessary to mitigate the spatial cognitive costs associated with teleporting, and landmarks were helpful only in the context of a geometric boundary.
\end{abstract}

Public Significance Statement: Movement through a virtual environment (VE) can be accomplished by walking, but physical space constraints require a navigation interface. One popular interface is teleporting, whereby the user points to a place in the VE and is instantly transported there. In this study, we reveal that teleporting can lead to spatial disorientation due to lack of self-motion cues. Boundaries in the VE, such as walls or a fence, reduce but do not eliminate the disorienting effect of teleporting. 
Keywords: Spatial cognition; Navigation; Triangle completion; Teleporting; Virtual reality 
Spatial cognitive implications of teleporting through virtual environments

Virtual reality (VR) is becoming increasingly popular in industry, education, and entertainment, partly due to the availability of low-cost systems such as the Oculus Rift and HTC Vive (Mainelli, Shirer, \& Ubrani, 2019). One especially compelling feature of most modern VR systems is the ability to explore virtual environments (VEs) by physically walking and turning, whereby cameras and accelerometers track the movement of the user within a finite tracked space, and the graphics in the head-mounted display (HMD) are updated based on the tracked movement. However, VEs commonly exceed the size of the walkable tracked space and therefore require a locomotion interface that allows for movement beyond that space. Perhaps the most common locomotion interface to allow for exploration of large VEs is teleportation (Figure 1), whereby the user selects a location on the ground plane and is immediately transported to that location without any self-motion cues.

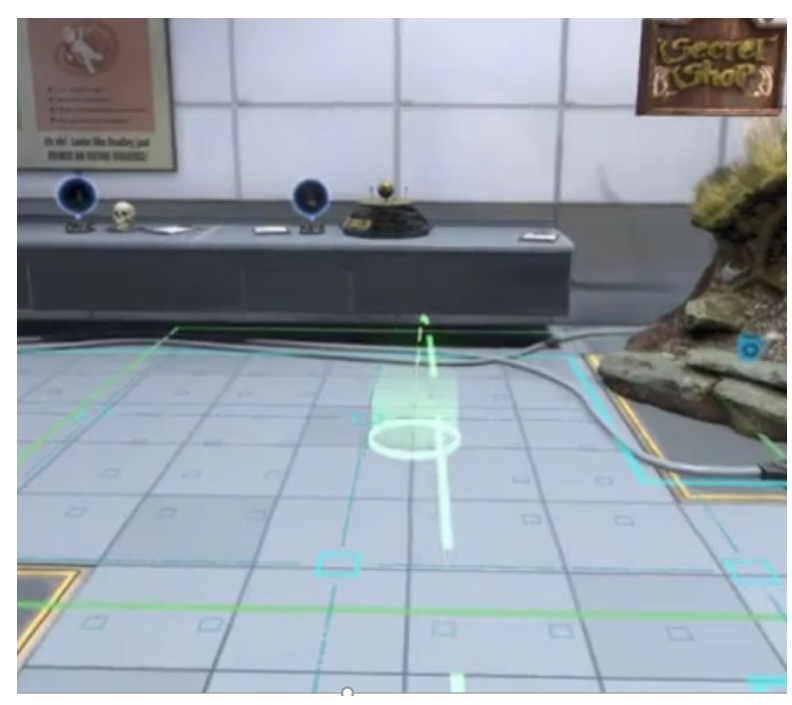

Figure 1. An example of the teleporting interface from a virtual reality game. From "The Lab," by Valve Corporation, 2016. Reprinted with permission. 
The teleportation interface is widespread in VR applications, most likely due to its ease of use (Bozgeyikli, Raij, Katkoori, \& Dubey, 2016; Langbehn, Lubos, \& Steinicke, 2018) and reduced likelihood of sickness compared with other interfaces, such as manipulating a joystick, that include visual self-motion without accompanying body motion (Christou \& Aristidou, 2017; Moghadam, Banigan, \& Ragan, 2018; Langbehn et al., 2018; Weißker, Kunert, Fröhlich, \& Kulik, 2018). Yet, the popularity of teleportation interfaces may come at a spatial cognitive cost. In particular, the lack of self-motion cues when teleporting may disrupt spatial updating, the process of keeping track of self-location during travel. Spatial updating failure can lead to disorientation (i.e., not knowing self-location relative to an external reference point or reference direction), which can be corrected only by using external piloting cues (e.g., landmarks) to reorient. Accurate spatial updating is critical for a successful VE for non-gaming purposes. Example applications include use of a VE for fire-fighter training aboard Navy ships (Tate, Sibert, \& King, 1997), training of electrical plant maintenance (De Sousa, Filho, Nunes, \& Lopes, 2010), virtual tourism (Go \& Gretzel, 2010), and construction site safety training (Moon, Becerik-Gerber, \& Soibelman, 2019). The current study investigated the influence of two common teleporting interfaces on spatial updating in VEs with and without piloting cues.

\section{Spatial Updating}

Spatial updating (updating self-location during travel) depends critically on self-motion cues, which can be broadly categorized as internal and external cues (see Chrastil \& Warren, 2012). Internal self-motion cues include vestibular stimulation, proprioception, and efference copies of motor commands, and are herein referred to collectively as body-based self-motion cues, or body-based cues for ease of exposition. Optic and acoustic flow that occur during selfmotion provide external self-motion cues. Spatial updating is informed by both types of self- 
motion cues, although research points to a particularly important role for body-based cues (Chance, Gaunet, Beall, \& Loomis, 1998; Grant \& Magee, 1998; Ruddle, Volkova, \& Bülthoff, 2011; Ruddle, Volkova, Mohler, \& Bülthoff, 2011; Waller, Loomis, \& Haun, 2004).

Triangle completion is a prototypical spatial updating task in which the participant moves along two legs of an outbound path before pointing to or directly returning to the path origin. In a foundational study (Klatzky, Loomis, Beall, Chance, \& Golledge, 1998), participants performed triangle completion under conditions that manipulated the availability of body-based and visual (optic flow) self-motion cues. Visual self-motion, when available, was provided through an HMD. Triangle completion error was greatest when movement was purely visual, comparatively small when participants physically walked and turned without vision, and equally small when participants physically rotated with accompanying visual rotation but received visual motion only when traveling along the two path legs. These results suggest that successful spatial updating requires body-based cues associated with rotation (change in orientation) and that bodybased cues associated with translation (change in position) are unnecessary.

In contrast to the findings reported by Klatzky et al. (1998), other research points to an important role for translational body-based cues. In one study (Ruddle \& Lessels, 2006), the researchers manipulated availability of body-based self-motion cues while participants performed a virtual foraging task. All conditions provided visual self-motion. Foraging errors were highest when locomotion was purely visual and lowest when locomotion involved walking and turning. In contrast to the results of Klatzky et al., errors when participants physically rotated but received only visual information about translation were just as high as in the purely visual condition, demonstrating an important role for body-based translational cues. These results 
indicate that successful spatial updating requires body-based cues associated with translation and rotation, and that rotational body-based cues alone are insufficient.

The role of optic flow in spatial updating appears smaller than that of body-based cues. Although triangle completion can sometimes be performed with optic flow alone (Riecke, van Veen, \& Bülthoff, 2002; but see Klatzky et al., 1998), spatial updating is worse with visual compared to body rotation (Wraga, Creem, \& Proffitt, 2004) and the influence of optic flow is minimal when body-based cues are also present (Kearns, Warren, Duchon \& Tarr, 2002).

To summarize, there is a general consensus in past research that body-based cues are important to spatial updating, although there is disagreement as to whether the critical contribution comes from rotational or translational body-based cues.

\section{Piloting}

Separate from the process of spatial updating is memory for locations, which are commonly stored relative to environmental cues such as landmarks, and guidance based on those landmarks is referred to as piloting. Proximal landmarks primarily provide information about position, whereas distal landmarks primarily provide information about direction (Padilla, Creem-Regehr, Stefanucci, \& Cashdan, 2017). Environmental shape, such as that defined by room walls, also provides a salient piloting cue (Kelly, McNamara, Bodenheimer, Carr, \& Rieser, 2008).

\section{Cue Combination}

Self-motion cues and piloting cues can be used together to improve spatial updating performance. Piloting can be used to zero out accumulated error in spatial updating (Zhang \& Mou, 2017), or can be combined with estimates from self-motion cues to reduce uncertainty about self-location (Chen, McNamara, Kelly, \& Wolbers, 2017; Sjolund, Kelly, \& McNamara, 
2018; Nardini, Jones, Bedford, \& Bradick, 2008). These findings indicate that spatial updating performance will suffer without body-based self-motion cues, even when rich landmarks are available to support piloting.

\section{Concordance: A Characteristic of Locomotion Interfaces for Virtual Environments}

The most natural way to explore a VE is by walking and turning one's body. In this case, movement through the VE is concordant with movement of the user's body, and thus all selfmotion cues are available to enable spatial updating (Figure 2, left panel). However, limited tracking space relative to the size of the VE necessitates a locomotion interface. The focus of the current study is on two implementations of the teleporting interface. The most common teleporting interface involves teleporting to translate and using the body to rotate. The user positions a marker on the ground plane and translates to that position without self-motion cues (visual or body-based), but rotates using the body and head. In this case, movement through the VE is partially concordant with movement of the user's body (Figure 2, middle panel). Other partially concordant interfaces include 1) peddling a stationary bike (Sun, Chan, \& Campos, 2004), whereby limb movements are consistent with movement through the VE, but vestibular cues indicate that the user is stationary, 2) scaled translational gain, whereby physical steps convert to larger virtual steps (Interrante, Ries, \& Anderson, 2007; Williams et al., 2006), and 3) redirected walking, whereby the user is redirected by modifying the relationship between rotation of the body and rotation through the VE, especially when the discrepancy is above 


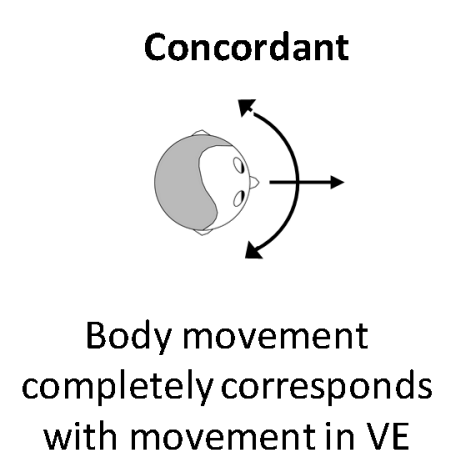

E.g., walking

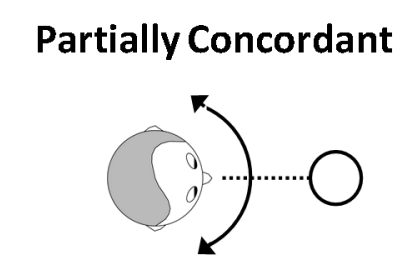

Body movement partially consistent with movement in VE

$$
\begin{aligned}
& \text { E.g., teleporting to } \\
& \text { translate only, riding a } \\
& \text { stationary bike }
\end{aligned}
$$

\section{Discordant}

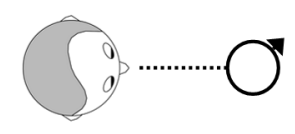

Body movement does not correspond to movement in VE

E.g., teleporting to
translate and rotate,
using a joystick to "fly"

Figure 2. The concordance framework, whereby locomotion interfaces are categorized based on the extent to which movement of the user's body corresponds with movement through the VE. Illustrations provide examples corresponding to the three interfaces used in the current study (left panel: user physically rotates and translates to move through the VE; middle panel: user physically rotates but teleports to translate; right panel: user teleports to translate and rotate).

threshold (Grechkin, Thomas, Azmandian, Bolas, \& Suma, 2016; Steinicke, Bruder, Jerald, Frenz, \& Lappe, 2010). Another implementation of the teleporting interface involves teleporting to translate and rotate through the VE, with no associated body movement. To rotate and translate, the user positions and orients a marker (e.g., an arrow) on the ground plane and teleports to that location and orientation. In this case, movement through the VE is discordant with movement of the user's body (Figure 2, right panel). Another discordant interface is joystick-based movement. However, such joystick interfaces are not favored due to their propensity to cause sickness (e.g., Christou \& Aristidou, 2017). In most VEs the user can switch between multiple interfaces (e.g., walking until reaching a physical boundary and switching to 
teleporting), and therefore locomotion is often accomplished through a combination of several interfaces varying in concordance.

Despite the popularity of teleporting interfaces for VR, there has been little research on the spatial cognitive consequences of teleporting. Existing research on the spatial cognitive effects of teleporting has compared teleporting with other locomotion interfaces that also lack body-based cues (Bowman, Koller, \& Hodges, 1997; Christou \& Aristidou, 2017; Langbehn et al., 2018; Moghadam et al., 2018; Weißker et al., 2018). Therefore, the unique contributions of the current project are to identify the spatial cognitive cost of teleporting with and without rotational body-based cues and to determine the impact of environmental cues, such as landmarks and spatial boundaries.

\section{Study Overview}

This study presents the results of five experiments that manipulated access to self-motion cues defined by three locomotion interfaces (walking, partially concordant teleporting, and discordant teleporting) while measuring triangle completion performance. Although past research (Klatzky et al., 1998; Ruddle \& Lessels, 2006) has included conditions similar to those tested here, those studies lacked key control conditions for making conclusions about popular VR interfaces. For example, the inference that body-based rotation is sufficient for accurate spatial updating is based on an imperfect comparison: Klatzky et al. tested a condition in which participants walked and rotated with the body but experienced no visual self-motion and a condition in which participants rotated with the body and experienced visual rotation and translation. In contrast, walking in VR includes body-based self-motion and visual self-motion, and partially concordant teleporting in VR includes body-based and visual rotation without translational self-motion cues. In other words, compared to the conditions tested by Klatzky et 
al., walking in VR might produce better performance than their walking condition, and partially concordant teleporting in VR might produce worse performance than their body rotation condition of Klatzky et al. The discordant teleporting interface might be similarly disadvantaged by the lack of visual self-motion, a condition that has not been previously tested. Thus, the current project is the first to include the locomotion conditions necessary for evaluating the spatial cognitive consequences of teleporting.

\begin{tabular}{lll} 
& Virtual environments & Research interest \\
\hline Experiment 1 & $\begin{array}{l}\text { Open field } \\
\text { Landmarks }\end{array}$ & Impact of landmarks (proximal + distal) \\
Experiment 2 & $\begin{array}{l}\text { Open field } \\
\text { Classroom }\end{array}$ & $\begin{array}{l}\text { Impact of orienting boundary (walls) with } \\
\text { landmarks (proximal only) }\end{array}$ \\
Experiment 3 & $\begin{array}{l}\text { Open field } \\
\text { Square fence + landmarks }\end{array}$ & $\begin{array}{l}\text { Impact of orienting boundary (fence) with } \\
\text { landmarks (proximal + distal) }\end{array}$ \\
Experiment 4 & $\begin{array}{l}\text { Square fence only } \\
\text { Square fence + landmarks }\end{array}$ & $\begin{array}{l}\text { Impact of landmarks (proximal + distal) } \\
\text { given orienting boundary }\end{array}$ \\
Experiment 5 & $\begin{array}{l}\text { Circular fence only } \\
\text { Circular fence }+ \text { landmarks }\end{array}$ & $\begin{array}{l}\text { Impact of landmarks (proximal }+ \text { distal) } \\
\text { given non-orienting boundary }\end{array}$
\end{tabular}

Table 1. Overview of the VEs used in each experiment, and the research interest that motivated the choice of VEs. Each experiment compared triangle completion performance in two environments when using three locomotion interfaces: walking, partially concordant teleporting, and discordant teleporting.

For applications using the teleporting interface, it is important to know whether environmental cues can mitigate the potentially negative effects of teleporting on spatial updating. Therefore, we also explored the influence of piloting cues, including landmarks and 
geometric (i.e., boundary shape) cues. Table 1 lists the environmental manipulations in each experiment.

\section{Experiment 1}

Experiment 1 evaluated the effects of interface concordance and piloting cues on spatial updating. Participants completed a triangle completion task in VR using three navigation interfaces: walking, partially concordant teleporting, and discordant teleporting. The VE was an endless grassy field with or without landmarks. We predicted that walking would produce the lowest errors, and the discordant condition would produce the highest errors across both VEs. Drawing inferences from similar conditions used by Klatzky et al. (1998), we predicted that partially concordant teleporting would produce performance on par with walking, although important differences across studies made this prediction speculative. We predicted that the landmarks VE would lead to lower triangle completion errors across all locomotion interfaces and that the beneficial effect of landmarks would be greatest when using the discordant teleporting interface.

Sample size was determined based on two related studies in which rotational and translational body-based cues were manipulated between participants (Klatzky et al., 1998; Ruddle \& Lessels, 2006). Comparisons which revealed a significant difference between locomotion conditions were calculated to have an average Cohen's d of 1.44, ranging from 1.0 to 2.0. Using the smallest reported effect size as a conservative estimate, a within-participant design would require 16 participants to achieve $95 \%$ power with alpha at .05 . Counterbalancing constraints required that participants be included by multiples of 12 . Therefore, all experiments had a sample size of 24 . 


\section{Method}

\section{Participants.}

Twenty-six students (9 men, 17 women) at Iowa State University participated in exchange for course credit. Data from two participants were removed (see Results) leaving 24 total participants ( 8 men, 16 women) in the dataset. The Iowa State Institutional Review Board approved the research protocols (IRB \#18-294).

\section{Hardware and software.}

The HTC Vive HMD displayed the VEs. A Windows 10 computer with an Intel 6700K processor and Nvidia GeForce GTX 1070 graphics card generated the graphics displayed in the Vive. Unity software displayed stereoscopic images at $1080 \times 1200$ resolution per eye with $100^{\circ}$ horizontal $\times 110^{\circ}$ vertical binocular field of view. Images refreshed at a rate of $90 \mathrm{~Hz}$ and reflected head position and orientation tracked by the Lighthouse tracking system sold with the Vive. One wireless handheld controller, also sold with the Vive, was used by participants to control the teleporting interfaces and to respond on each trial.

\section{Stimuli.}

Videos showing the triangle completion task with each interface and in each environment are available on the Open Science Framework: https://osf.io/m4zfv//. The open field VE (Figure 3, top-left) consisted of an infinite ground plane with grass texture and uniform blue sky. The landmarks VE (Figure 3, top-middle) included proximal and distal landmarks in addition to the grass field with blue sky. Proximal landmarks (e.g., plants, trees, and bench) were located near the intended triangle paths but were placed far enough away to not be directly on the path. Distal landmarks (bridge, building, mountains, and arch) were placed in the far distance and spaced every $90^{\circ}$. 
Semi-transparent vertical posts, each $1 \mathrm{~m}$ tall and $.25 \mathrm{~m}$ in diameter marked the path used to perform the triangle completion task. A green post marked the beginning of the path, a yellow post marked the end of the first path leg, and a red post marked the end of the second path $\operatorname{leg}^{1}$. Green post locations were located in a ring around the center of the environment. The yellow post led the participant toward the center of the environment, and the red post led the participant away from the center of the environment. At the base of each post was a white arrow with a height of $7.5 \mathrm{~cm}$ (Figure 3, top-left panel), which indicated the direction of the next post. The arrows were necessary to indicate the intended facing direction when using the discordant teleporting interface and were present when using all locomotion interfaces. The arrow at the base of the green post pointed in the direction of the yellow post, the arrow at the base of the yellow post pointed in the direction of the red post, and the arrow at the base of the red post pointed in the same direction as the arrow on the yellow post.

\footnotetext{
${ }^{1}$ Participants were not screened for color vision, but post color was redundant with post sequence and therefore was not an essential feature. No participants reported difficulty with viewing the stimuli.
} 

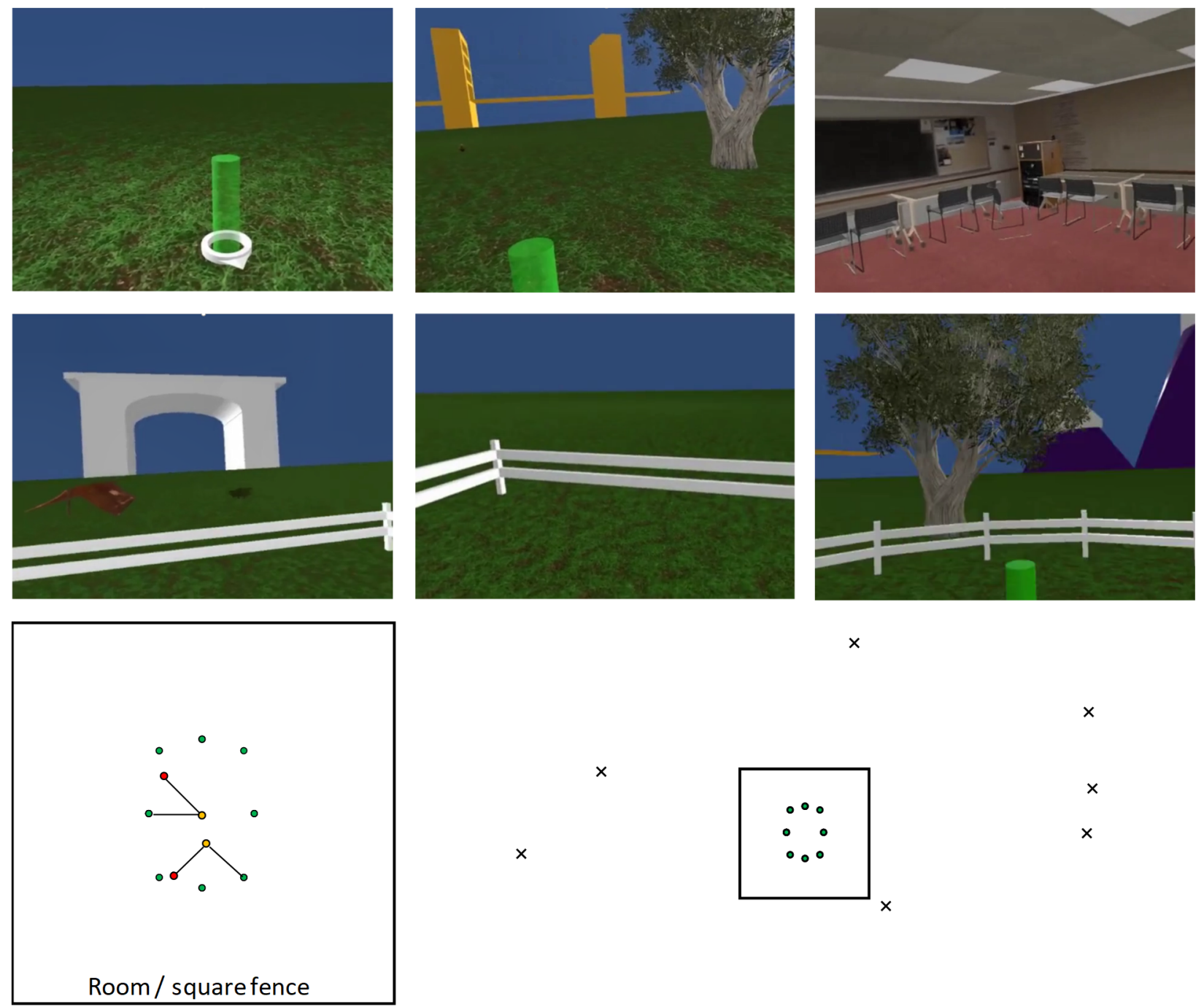

Room / square fence

Figure 3. Virtual environments used in Experiments 1-5. Top row from left to right shows the open field (Exp 1, 2, and 3), landmarks (Exp 1), and classroom (Exp 2) environments. Middle row from left to right shows the square fence + landmarks (Exp 3 and 4), square fence only (Exp 4), and the circular fence + landmarks (Exp 5) environment. Bottom row shows overhead views of the VEs including possible post locations, boundaries, and landmarks (right panel only).

A virtual replica of the handheld controller was visible at all times, indicating the position and orientation of the actual controller. The partially concordant teleporting interface was controlled by positioning a white circle $(30 \mathrm{~cm}$ diameter) with surrounding white ring $(75 \mathrm{~cm}$ 
diameter) in the intended location on the ground plane (Figure 4, left). A thin red line extended from the joystick to the center of the white circle. The participant pressed and held the trackpad on the top of the controller while manipulating the location of the teleport marker by pointing with the controller. Releasing the trackpad teleported the participant to the selected location (orientation was unchanged). The discordant teleporting interface was controlled by positioning and orienting a magenta ring (height: $7.5 \mathrm{~cm}$; outer diameter: $195 \mathrm{~cm}$ ) with an arrow on one side (Figure 4, right). A thin red line extended from the joystick to the center of the ring. The participant pressed and held the trackpad button to bring up the teleporting ring, and rotated the ring by moving the thumb around the edge of the circular trackpad. Releasing the trackpad button teleported the participant to the selected location and orientation.
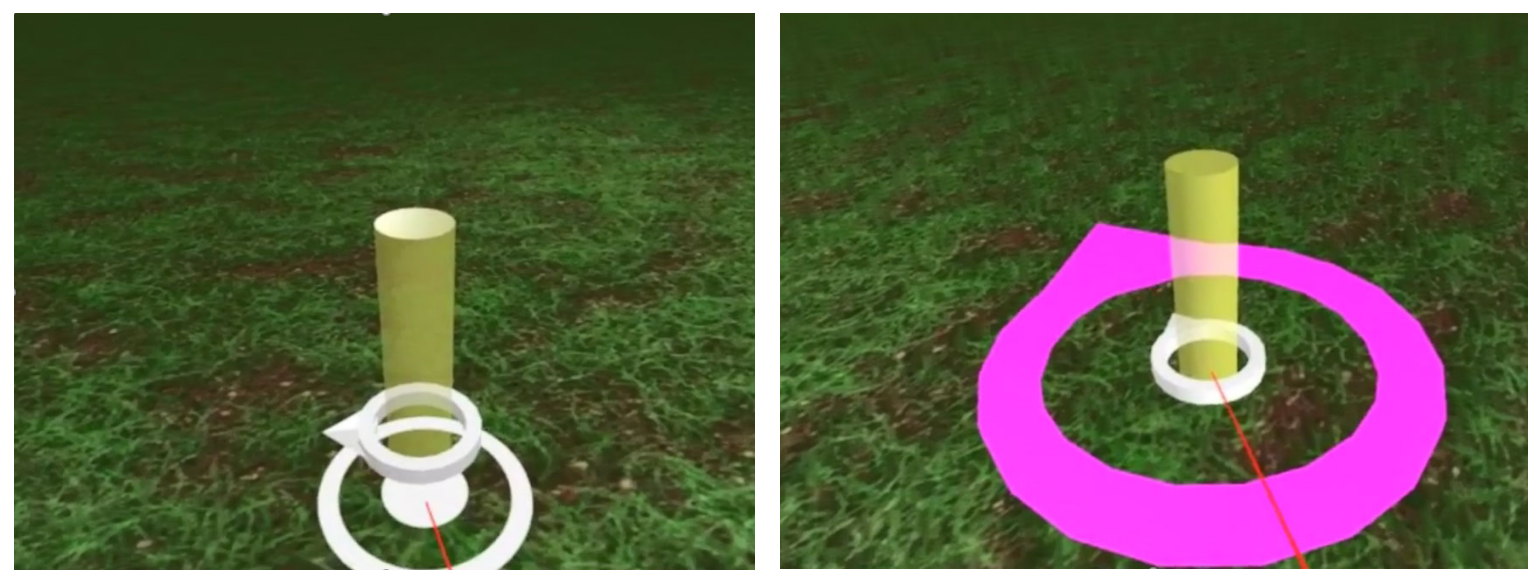

Figure 4. Screenshots taken from the participant's perspective while using the partially concordant teleporting interface (left) and the discordant teleporting interface (right). 


\section{Design.}

The experiment employed a 2 (VE: open field or landmarks) by 3 (interface: walking, partially concordant teleporting, or discordant teleporting) repeated-measures design. For each combination of VE and interface, participants performed a block of 12 triangle completion trials corresponding to 12 unique turn angles spaced every $22.5^{\circ}$ from $-135^{\circ}$ to $+135^{\circ}$. Path leg length

was randomly selected on each trial from three possible values for the first and second leg (1.52, 1.68 , or $1.83 \mathrm{~m})$. There were eight possible locations of the path origin chosen to ensure that the participant would not navigate outside of the tracked space when using the walking interface. The first path leg led participants toward the center of the environment, and the second path leg led participants away from the center of the environment. Origin location was pseudorandomized across trials with the constraint that the same origin location was not repeated on sequential trials.

The VE variable was blocked, such that three blocks corresponding to the three locomotion interfaces were completed first in one VE and then in the other VE. VE order was counterbalanced. Interface order was also counterbalanced, and the same order was used for both VE blocks. Pointing location and pointing response time was recorded. Sessions lasted up to one hour.

\section{Procedure.}

After signing the informed consent, the participant received verbal instructions on the triangle completion task. The participant then donned the HMD and was trained on performing the triangle completion task with each of the three interfaces. Training took place in a VE with a grid-like ground texture and no landmarks. The participant was required to perform three 
practice triangle completion trials with each locomotion interface and could request additional practice. Experimental trials began after completion of practice.

A green post marking the path origin appeared at the beginning of each trial. The participant traveled to the green post, which disappeared upon arrival and a yellow post appeared, marking the first leg of the path. Upon arrival at the yellow post it disappeared and a red post appeared, marking the second leg of the path. Upon arrival at the red post, it disappeared, and the participant was prompted to point to the remembered location of the path origin. Pointing was accomplished by positioning a blue circle $(38 \mathrm{~cm}$ diameter $)$ on the ground plane. A thin red line extended from the joystick to the center of the blue circle. The participant pressed and held the trigger button located on the controller while manipulating the location of the blue circle and the experimenter then pressed a key to log the participant's response and advance to the next trial. Feedback was never provided.

\section{Results}

Absolute error was calculated as the absolute distance (in meters) between the path origin and pointing response location. Absolute error could be caused by angular error (pointing in the wrong direction), radial error (pointing the wrong distance), or some combination. Separate analyses of angular and radial error revealed similar patterns across manipulations of interface and environment. For brevity, we report only absolute error, which combines both angular and radial error components. Response latency was calculated as the difference between the time when the participant arrived at the red post and when a pointing response was recorded.

Data from one participant were removed due to incomplete data (at least one missing cell in the experimental design, attributable to experimenter error or failure to complete the study). Data from another participant were removed due to mean pointing errors that were more than 
three standard deviations higher than the group mean. An additional 38 trials $(2.2 \%)$ were removed from the remaining data due to computer errors and procedural errors.

Analyses focused on the effects of interface and environment. Therefore, data from repeated trials for each environment and interface were averaged together prior to analysis. There was no evidence of speed-accuracy tradeoff. The within-participant correlation between error and latency was significantly positive $(M=.47, S E=.09), t(23)=5.29, p<.001$. Pointing error was the focus of the current project, and it was generally more responsive to manipulation of the independent variables than was response latency. Latency results are provided on the Open Science Framework: https://osf.io/m4zfv/.

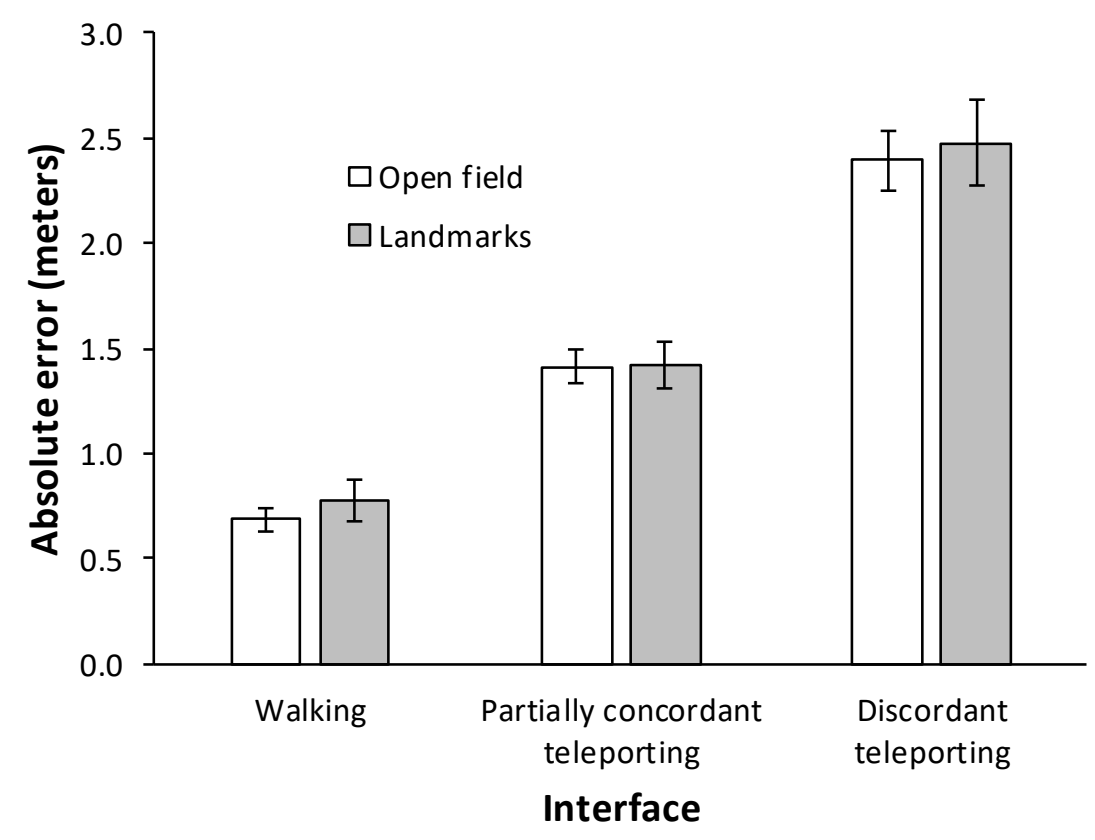

Figure 5. Average absolute error when performing the triangle completion task in Experiment 1. Error bars represent +/- 1 SEM.

Absolute errors (Figure 5) were analyzed in a repeated measures ANOVA with terms for interface and environment. Only the main effect of interface was significant, $F(2,46)=114.19, p$ 
$<.001, \eta_{p}{ }^{2}=.83$. The main effect of environment was not significant $F(1,23)=.60, p=.45, \eta_{p}^{2}=$ .03 , nor was the interaction between interface and environment, $F(2,46)=.17, p=.84, \eta_{p}^{2}=.01$. Errors in the walking interface $(M=0.73, S E=0.07)$ were significantly lower than those in the partially concordant teleporting interface $(M=1.42, S E=0.09), t(23)=8.51, p<.001, d=1.75$, and errors in the partially concordant teleporting interface were significantly lower than those in the discordant teleporting interface $(M=2.44, S E=0.16), t(23)=8.12, p<.001, d=1.66$.

\section{Discussion}

Spatial updating performance was best with the walking interface, worst with the discordant teleporting interface, and in between with the partially concordant teleporting interface. These results are consistent with the notion that body-based cues play an important role in spatial updating: the partially concordant teleporting interface lacked translational bodybased cues, and the discordant teleporting interface lacked translational and rotational bodybased cues. This pattern of results seems logical, yet is somewhat inconsistent with past research on the role of body-based cues in spatial updating (Klatzky et al., 1998). The divergent findings are considered in detail in the General Discussion.

The more surprising result is that landmarks did not improve spatial updating performance compared to performance in the open field VE. Landmarks were expected to improve spatial updating with all interfaces, but particularly with the teleporting interfaces due to the lack of body-based cues. Past work indicates that body-based cues are integrated with landmark cues when walking (Chen et al., 2017; Nardini et al., 2008). However, those studies included landmarks that were relatively close to the target location, thereby providing both positional and directional information. It is possible that the landmarks in Experiment 1 were sufficiently far away as to be useful only as directional cues, although this still does not explain 
why they were not helpful at all. Another factor is that the HMD limits the horizontal field of view to $100^{\circ}$, thereby limiting the possibility of using a collection of several landmarks to triangulate position.

\section{Experiment 2}

The landmarks VE from Experiment 1 did not facilitate spatial updating compared to the open field VE. Therefore, Experiment 2 utilized an indoor virtual environment composed of landmarks as well as walls, which provide a salient geometric cue to self-location (Sjolund et al., 2018). Although the differences between the landmarks VE and the classroom VE are so numerous that they defy succinct description, the landmarks VE was considered again later in Experiment 3 in light of insights gained through the classroom VE in Experiment 2. Research on spatial memory points to an important role for room shape in defining the reference frame used to organize remembered object locations (Kelly \& McNamara, 2008; Shelton \& McNamara, 2001). Furthermore, animal neuroscience has identified neurons that respond primarily to boundaries (Hartley, Burgess, Lever, Cacucci, \& O’Keefe, 2000; Lever, Burton, Jeewajee, O'Keefe, \& Burgess, 2009), and it is believed that these "boundary vector cells" provide key inputs into the animal's representation of self-location (O'Keefe \& Burgess, 1996; Burgess, Jackson, Hartley, \& O’Keefe, 2000).

Participants completed the triangle completion task using the same three navigation interfaces from Experiment 1: walking, partially concordant teleporting, and discordant teleporting. The VE was a classroom or open field. On the basis of the Experiment 1 results, errors were predicted to be lowest for walking, largest for discordant teleporting, and in between for partially concordant teleporting, regardless of VE. Furthermore, errors were predicted to be 
lower in the classroom VE compared to the open field VE, and that the difference between VE would be exaggerated when teleporting compared to walking.

\section{Methods}

\section{Participants.}

Twenty-seven students (13 men, 14 women) at Iowa State University participated in exchange for course credit. Data from three participants were removed (see Results) leaving 24 total participants (13 men, 11 women) in the dataset.

\section{Stimuli, design, and procedure.}

The classroom VE (Figure 3, top-right) was designed based on a real classroom at Iowa State University. The walls of the 3D model were textured with photographs from the real classroom. The classroom VE was square with 11-meter sides, and included several 3D models of classroom furniture such as chairs, tables, and a classroom media console (furniture and other virtual objects were placed near the perimeter of the room to ensure that the participant did not collide with virtual objects while performing the triangle completion task).

The experiment employed a 2 (VE: open field or classroom) by 3 (interface: walking, partially concordant teleporting, or discordant teleporting) repeated-measures design. The experimental design otherwise followed the Experiment 1 design.

\section{Results}

Data from three participants were removed due to incomplete data (at least one missing cell in the experimental design; attributable to experimenter error or failure to complete the study in the allotted time). An additional 26 trials (1.5\%) were removed from the remaining data due to computer errors and procedural errors. 
There was no evidence of speed-accuracy tradeoff. The within-participant correlation between error and latency was significantly positive $(M=.47, S E=.09), t(23)=5.30, p<.001$.

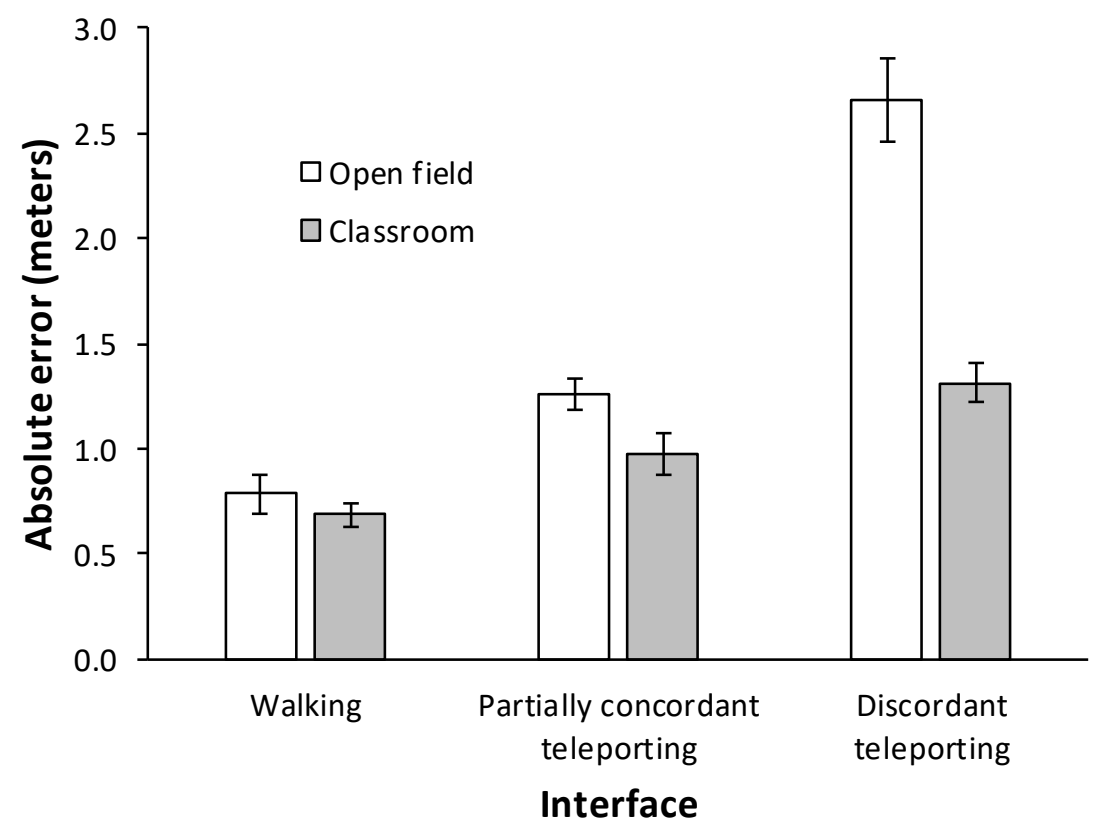

Figure 6. Average absolute error when performing the triangle completion task in Experiment 2. Error bars represent +/- 1 SEM.

Absolute errors (Figure 6) were analyzed in a repeated measures ANOVA with terms for interface and environment. Significant main effects of interface, $F(2,46)=67.93, p<.001, \eta_{p}{ }^{2}=$ .75 , and environment, $F(1,23)=38.31, p<.001, \eta_{p}^{2}=.63$, were qualified by a significant interaction between interface and environment, $F(2,46)=31.89, p<.001, \eta_{p}^{2}=.58$. In each environment, the discordant teleporting interface produced larger errors than the partially concordant teleporting interface (open field: $t(23)=7.08, p<.001, d=1.44$; classroom: $t(23)=$ $3.65, p=.001, d=.74$ ), and the partially concordant teleporting interface produced larger errors than the walking interface (open field: $t(23)=5.74, p<.001, d=1.24$; classroom: $t(23)=2.91, p$ $=.008, d=.59)$. Furthermore, the classroom produced lower errors compared to the open field 
when using the partially concordant teleporting interface $t(23)=2.37, p=.03, d=.48$, and the discordant teleporting interface $t(23)=7.59, p<.001, d=1.55$, but not when using the walking interface, $t(23)=1.05, p=.30, d=.20$.

\section{Discussion}

Consistent with Experiment 1 results, spatial updating performance was best with the walking interface, worst with the discordant teleporting interface, and in between with the partially concordant teleporting interface. This pattern of results was found in the open field VE and the classroom VE. However, spatial updating performance in the classroom VE was better than in the open field VE when using the partially concordant and discordant teleporting interfaces. In other words, removal of body-based cues in the two teleporting interfaces negatively affected spatial updating in both VEs, but to a lesser extent in the classroom VE than in the open field VE. This result is in stark contrast to the landmarks VE in Experiment 1, which did not facilitate spatial updating performance with any of the three interfaces.

Why did the classroom VE, but not the landmarks VE, reduce spatial updating errors when using the two teleporting interfaces? Both VEs contained seemingly ample landmarks, although the landmarks differed in several ways, including proximity to the navigation space. One distinction between the VEs is that the classroom VE contained a boundary formed by four walls whereas the landmarks VE had no such boundary. Geometric cues defined by room walls affect human spatial memory (Kelly \& McNamara, 2008; Shelton \& McNamara, 2001) and are integrated with self-motion cues during human spatial updating (Kelly et al., 2008; Sjolund et al., 2018). Furthermore, animal research has identified neurons that respond primarily to geometric boundaries (Lever et al., 2009). 


\section{Experiment 3}

Experiment 3 was designed to determine whether the landmarks VE from Experiment 1 would facilitate spatial updating if it also contained a geometric boundary. Therefore, Experiment 3 added a square fence to the landmarks VE used in Experiment 1. The dimensions of the fence were the same as those of the classroom used in Experiment 2, and the fence style was chosen to allow a clear view of the landmarks beyond the fence. Spatial updating performance in this "fence + landmarks VE" was compared with performance in the open field VE. Based on the previous experiments, errors were predicted to be lowest for walking, largest for discordant teleporting, and in between for partially concordant teleporting, regardless of VE. No explicit predictions were made as to whether the fence + landmarks VE would facilitate performance due to the discrepant effect of the landmarks VE (Exp 1) and the classroom VE $(\operatorname{Exp} 2)$

\section{Methods}

\section{Participants.}

Twenty-eight students (11 men, 17 women) at Iowa State University participated in exchange for course credit. Data from four participants were removed (see Results) leaving 24 total participants (11 men, 13 women) in the dataset.

\section{Stimuli, design, and procedure.}

The fence + landmarks VE (Figure 3, middle-left) was identical to the landmarks VE from Experiment 1 except for the addition of a large square fence. Landmark locations were unchanged from Experiment 1, and all landmarks fell outside of the fence boundaries. Each side of the fence consisted of two horizontal rails and four vertical posts. 
The experiment employed a 2 (VE: open field or fence + landmarks) by 3 (interface: walking, partially concordant teleporting, or discordant teleporting) repeated-measures design. The experimental design otherwise followed the design of the prior experiments.

\section{Results}

Data from four participants were removed due to incomplete data (at least one missing cell in the experimental design; attributable to experimenter error or failure to complete the study in the allotted time). An additional 35 trials (2.0\%) were removed from the remaining data due to computer errors and procedural errors.

There was no evidence of speed-accuracy tradeoff. The within-participant correlation between error and latency was significantly positive $(M=.50, S E=.07), t(23)=6.86, p<.001$. Absolute errors (Figure 7) were analyzed in a repeated measures ANOVA with terms for interface and environment. Significant main effects of interface, $F(2,46)=118.21, p<.001, \eta_{p}^{2}=$ .84 , and environment, $F(1,23)=33.72, p<.001, \eta_{p}^{2}=.60$, were qualified by a significant interaction between environment and interface, $F(2,46)=8.87, p=.001, \eta_{p}^{2}=.28$. In each environment, the discordant teleporting interface produced larger errors than the partially concordant teleporting interface (open field: $t(23)=6.05, p<.001, d=1.24$; fence + landmarks: $t(23)=4.31, p<.001, d=.89)$, and the partially concordant teleporting interface produced larger errors than the walking interface (open field: $t(23)=6.11, p<.001, d=1.23$; fence + landmarks: $t(23)=8.70, p<.001, d=1.78)$. Furthermore, the fence + landmarks VE produced lower errors compared to the open field when using the partially concordant teleporting interface, $t(23)=$ 2.29, $p=.03, d=.45$, and the discordant teleporting interface, $t(23)=4.26, p<.001, d=.87$, but not when using the walking interface, $t(23)=1.22, p=.24, d=.26$ 


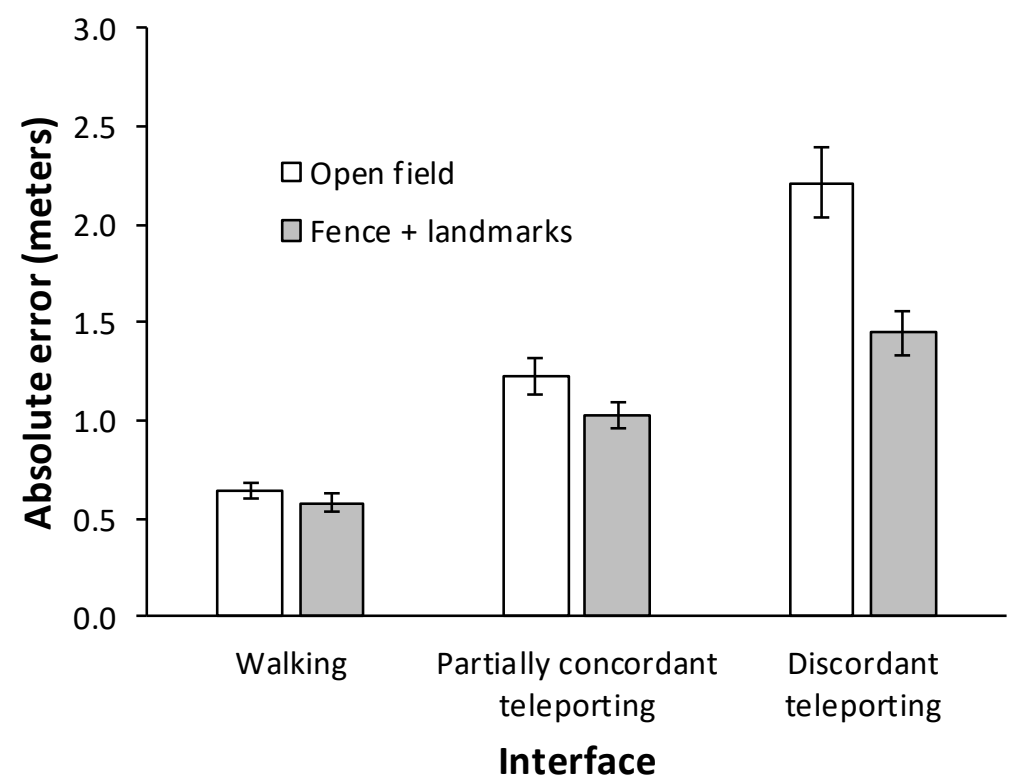

Figure 7. Average absolute error when performing the triangle completion task in Experiment 3. Error bars represent +/- 1 SEM.

\section{Discussion}

Consistent with results from Experiments 1 and 2 spatial updating performance was best with the walking interface, worst with the discordant teleporting interface, and in between with the partially concordant teleporting interface. Although this pattern of results was found in both the open field VE and the fence + landmarks VE, spatial updating performance in the fence + landmarks VE was better than in the open field VE when using the partially concordant and discordant teleporting interfaces. The facilitative influence of the fence + landmarks VE stands in stark contrast to the landmarks VE in Experiment 1, which did not facilitate spatial updating performance with any of the three interfaces. Therefore, the presence of a rectangular boundary may be the critical distinction between the landmarks VE in Experiment 1 and the classroom VE in Experiment 2. 
The fence may have led participants to use the landmarks; it is also possible that participants did not use the landmarks and instead relied exclusively on the square fence in the fence + landmarks VE. The square fence alone is a visual cue to self-orientation, but it is somewhat ambiguous due to its rotational symmetry (visual features of the square fence repeat every $90^{\circ}$ ). That is, the square fence is an informative cue when the navigator can determine selforientation within $+/-45^{\circ}$, such that a navigator who is uncertain about self-orientation by a relatively small amount can use the fence to zero out accumulated error. But if uncertainty exceeds that threshold, the navigator will not know which side of the fence he/she is facing, and therefore the fence's value will be lost.

\section{Experiment 4}

In order to examine the sufficiency of the square boundary in the fence + landmarks VE from Experiment 3, Experiment 4 evaluated spatial updating performance in a "fence only VE," which contained the same fence used in the fence + landmarks VE but without the landmarks. Performance in the fence only VE was compared to the fence + landmarks VE. Triangle completion errors were predicted to be lowest for walking, largest for discordant teleporting, and in between for partially concordant teleporting, regardless of VE. No predictions were made as to whether the fence only VE would facilitate performance to the same extent as the fence + landmarks VE.

\section{Methods}

\section{Participants.}

Twenty-nine students (15 men, 14 women) at Iowa State University participated in exchange for course credit. Data from five participants were removed (see Results) leaving 24 total participants (12 men, 12 women) in the dataset. 


\section{Stimuli, design, and procedure.}

The fence only VE was created by removing all of the landmarks from the fence + landmarks VE, leaving only the fence and grassy field.

The experiment employed a 2 (VE: fence only or fence + landmarks) by 3 (interface: walking, partially concordant teleporting, or discordant teleporting) repeated-measures design. The experimental design otherwise followed the design of the prior experiments.

\section{Results}

Data from five participants were removed due to incomplete data (at least one missing cell in the experimental design; attributable to experimenter error or failure to complete the study in the allotted time). An additional 33 trials (1.91\%) were removed from the remaining data due to computer errors and procedural errors.

There was no evidence of speed-accuracy tradeoff. The within-participant correlation between error and latency was significantly positive $(M=.57, S E=.06), t(23)=9.89, p<.001$. Absolute errors (Figure 8) were analyzed in a repeated measures ANOVA with terms for interface and environment. The main effect of interface was significant, $F(2,46)=48.96, p<$ $.001, \eta_{p}^{2}=.68$, as was the interaction between environment and interface, $F(2,46)=4.72, p=$ $.01, \eta_{p}^{2}=.17$. The main effect of environment was not significant, $F(1,23)=2.02, p=.17$. In each environment, the discordant teleporting interface produced larger errors than the partially concordant teleporting interface (fence only: $t(23)=6.22, p<.001, d=1.27$; fence + landmarks: $t(23)=4.25, p<.001, d=.88)$, and the partially concordant teleporting interface produced larger errors than the walking interface (fence only: $t(23)=5.37, p<.001, d=1.08$; fence + landmarks: $t(23)=4.60, p<.001, d=.93)$. Furthermore, the fence + landmarks VE produced lower errors compared to the fence only VE when using the discordant teleporting interface $t(23)=2.17, p=$ 
$.04, d=.43$, but not when using the partially concordant teleporting interface and the walking interface (partially concordant teleporting: $t(23)=.33, p=.75, d=.07$; walking: $t(23)=.81, p=$ $.43, d=.16)$.

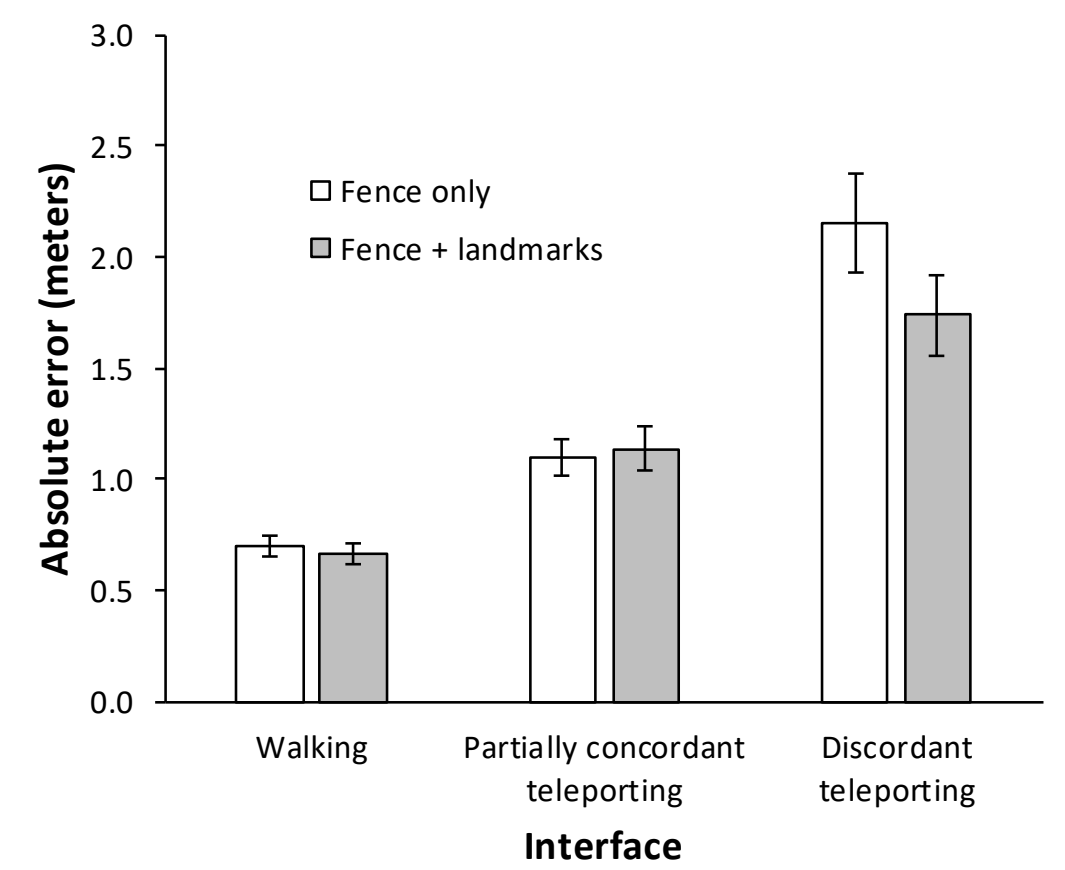

Figure 8. Average absolute error when performing the triangle completion task in Experiment 4. Error bars represent +/- 1 SEM.

\section{Discussion}

Consistent with results from Experiments 1-3, spatial updating performance was best with the walking interface, worst with the discordant teleporting interface, and in between with the partially concordant teleporting interface. Although this pattern of results was found in both the fence + landmarks VE and the fence only VE, spatial updating performance in the fence + landmarks VE was better than in the fence only VE when using the discordant teleporting interface. These results indicate that a boundary without landmarks can be sufficient and that additional landmarks are not necessary when all body-based cues are available (walking interface) or when only rotational body-based cues are available (partially concordant teleporting 
interface), but that landmarks visible beyond the boundary improve spatial updating performance when no body-based cues are available (discordant teleporting interface).

Why were landmarks (in addition to the fence) helpful only when using the discordant teleporting interface? In order for the square fence to be useful by itself, the navigator must be able to determine self-orientation within $+/-45^{\circ}$ due to the rotational symmetry of the square fence (see Experiment 3 discussion for more detailed discussion). Therefore, one explanation for why landmarks were helpful only with the discordant teleporting interface is that participants were generally able to determine self-orientation within $+/-45^{\circ}$ when using the partially concordant teleporting interface, but at least occasionally exceeded that threshold when using the discordant teleporting interface. One implication for future research is that an unambiguous boundary should lead to equivalent spatial updating performance whether or not landmarks are included.

\section{Experiment 5}

The fence + landmarks VE from Experiment 3 facilitated spatial updating compared to the open field VE when navigating with the teleporting interfaces, in contrast to the landmarks VE from Experiment 1. We speculate that the value of landmarks in this context is that they define a boundary's orientation. If true, then even a circular boundary should benefit from added landmarks beyond the boundary. This would provide particularly strong support of the theory since landmarks alone (Experiment 1) did not facilitate spatial updating, and a circular fence alone should also not be especially useful. Experiment 5 compared spatial updating performance with a circular boundary only ("circle only VE") and a circular boundary with the same landmarks used in Experiment 1 ("circle + landmarks VE"). 


\section{Methods}

\section{Participants.}

Twenty-eight students (10 men, 18 women) at Iowa State University participated in exchange for course credit. Data from four participants were removed (see Results) leaving 24 total participants (9 men, 15 women) in the dataset.

\section{Stimuli, design, and procedure.}

The circle + landmarks VE (Figure 3, middle-right) used the same proximal and distal landmarks from the landmarks VE condition in Experiment 1 and added a circular fence $(11.3 \mathrm{~m}$ diameter). The circle only VE was created by removing all of the landmarks from the circle + landmarks VE, leaving only the circular fence and grassy field.

The experiment employed a 2 (VE: circle only or circle + landmarks) by 3 (interface: walking, partially concordant teleporting, or discordant teleporting) repeated-measures design. The experimental design otherwise followed the design of the prior experiments.

\section{Results}

Data from four participants were removed due to incomplete data (at least one missing cell in the experimental design; attributable to experimenter error or failure to complete the study in the allotted time). An additional 39 trials (2.26\%) were removed from the remaining data due to computer errors and procedural errors.

There was no evidence of speed-accuracy tradeoff. The within-participant correlation between error and latency was significantly positive $(M=.60, S E=.07), t(23)=8.08, p<.001$. 


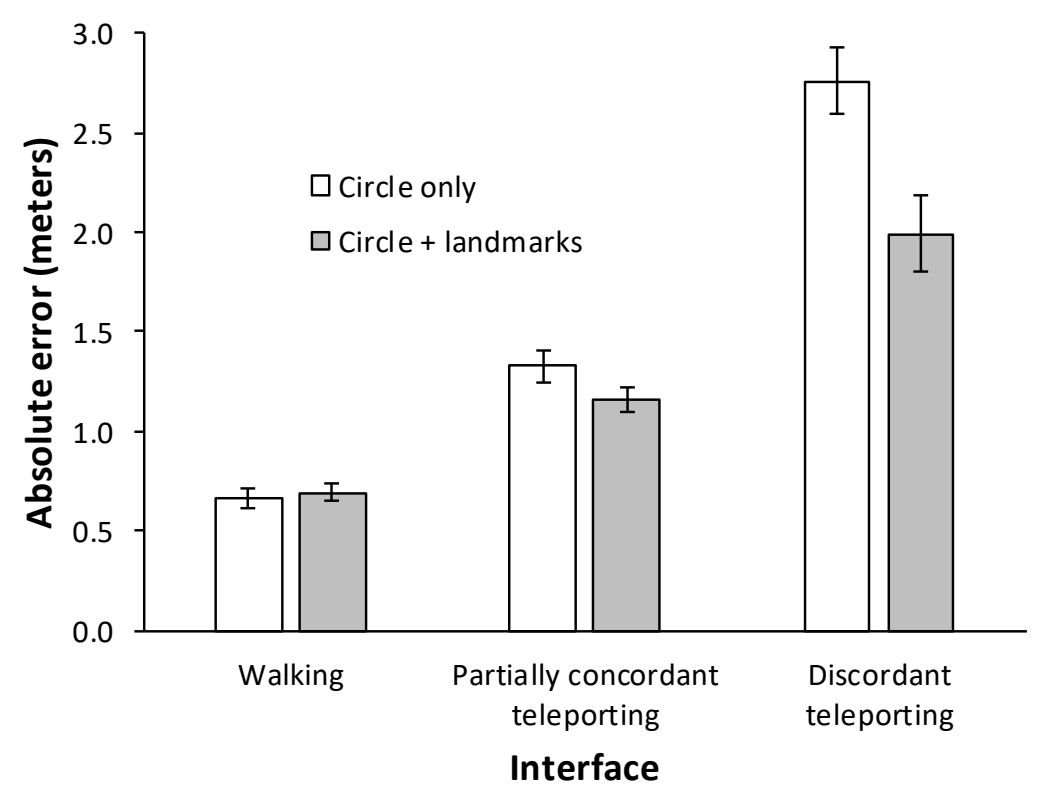

Figure 9. Average absolute error when performing the triangle completion task in Experiment 5. Error bars represent +/- 1 SEM.

Absolute errors (Figure 9) were analyzed in a mixed model ANOVA with terms for interface and environment. Significant main effects of interface, $F(2,46)=121.07, p<.001, \eta_{p}^{2}$ $=.84$, and environment $F(1,23)=12.62, p=.002, \eta p^{2}=.35$, were qualified by a significant interaction between environment and interface, $F(2,46)=7.84, p=.001, \eta_{p}^{2}=.25$. In each environment, the discordant teleporting interface produced larger errors than the partially concordant teleporting interface (circle only: $t(23)=7.85, p<.001, d=1.61$; circle + landmarks: $t(23)=4.70, p<.001, d=.95)$, and the partially concordant teleporting interface produced larger errors than the walking interface (circle only: $t(23)=7.66, p<.001, d=1.57$; circle + landmarks: $t(23)=6.38, p<.001, d=1.31)$. The circle + landmarks VE produced lower errors compared to the circular fence when using the discordant teleporting interface, $t(23)=3.29, p=.003, d=.67$, and the same pattern was found when using the partially concordant teleporting interface but the 
difference did not reach statistical significance, $t(23)=1.80, p=.08, d=.36$. There was no benefit to the added landmarks when using the walking interface, $t(23)=.65, p=.52, d=.14$.

\section{Discussion}

Compared to the circle only, the circle + landmarks VE led to better spatial updating performance when using the discordant teleporting interface and the partially concordant teleporting interface, although the latter difference was marginally significant. These data support the theory that landmarks are especially valuable when they can be used to provide orientation to a boundary and stand in contrast to the finding from Experiment 1 that landmarks without a boundary do not improve performance. This is considered further in the General Discussion.

\section{General Discussion}

The primary goals of this project were to evaluate whether there are spatial cognitive costs associated with the use of two teleporting interfaces commonly found in virtual reality applications and to identify environmental cues that could mitigate those costs. Participants completed a triangle completion task using three locomotion interfaces: 1) walking, which included body-based and visual self-motion cues, 2) partially concordant teleporting, which included body-based and visual rotational cues but no translational cues, and 3) discordant teleporting, which lacked all self-motion cues. Across five experiments, discordant teleporting produced larger errors than partially concordant teleporting, which in turn produced larger errors than walking. Furthermore, geometric boundaries (room walls or a fence) were necessary to mitigate the spatial cognitive costs associated with teleporting, and landmarks alone were unhelpful (see Table 2 for summary of results from individual experiments). 
Virtual environments

\begin{tabular}{|c|c|}
\hline Experiment 1 & $\begin{array}{l}\text { Open field } \\
\text { Landmarks }\end{array}$ \\
\hline Experiment 2 & $\begin{array}{l}\text { Open field } \\
\text { Classroom }\end{array}$ \\
\hline Experiment 3 & $\begin{array}{l}\text { Open field } \\
\text { Square fence }+ \text { landmarks }\end{array}$ \\
\hline
\end{tabular}

Experiment 4 Square fence only

Square fence + landmarks

Experiment 5 Circular fence only

Circular fence + landmarks

\section{Main findings}

Errors did not differ across VEs.

Lower errors in the classroom for partially concordant and discordant teleporting compared to open field.

Walking errors did not differ across virtual environments.

Lower errors in the square fence + landmarks for partially concordant and discordant teleporting compared to open field.

Walking errors did not differ across virtual environments.

Lower errors in the square fence + landmarks for discordant teleporting.

Walking and partially concordant teleporting did not differ across virtual environments.

Lower errors in the circular fence + landmarks for discordant teleporting.

Walking and partially concordant teleporting did not differ across virtual environments.

Table 2. Summary of main findings across all experiments. In all environments, errors were larger with discordant teleporting than partially concordant teleporting, and larger with partially concordant teleporting than walking. 
Based on past work suggesting that body-based rotational cues are both necessary and sufficient for triangle completion (Klatzky et al., 1998), it was predicted that walking and partially concordant teleporting would produce equally low triangle completion errors compared to discordant teleporting. The actual pattern observed across the five experiments reported here is only somewhat consistent with the results of Klatzky et al. (1998), with the main departure being the larger errors with the partially concordant teleporting interface compared to the walking interface. However, there were several methodological differences across studies. For example, 1) the partially concordant and discordant teleporting interfaces in the current study did not provide translational optic flow, 2) the walking interface in the current study included bodybased and visual self-motion cues, 3) all conditions in the current study involved active selection of locomotion destination, and 4) all conditions in the current study provided information about the turn angle prior to executing the turn (i.e., the arrow on the yellow post). Two of these differences stand out as possibly increasing the difference in performance between partially concordant teleporting and walking, specifically the absence of translational optic flow in partially concordant teleporting and the presence of optic flow in the walking interface. A future experiment in which the partially concordant interface is tested with and without translational optic flow could help answer this question, but it is not practical in an applied context due to the increased sickness when translational optic flow is provided in the absence of translational body movement (Christou \& Aristidou, 2017; Moghadam et al., 2018; Langbehn et al., 2018; Weißker et al., 2018).

It was also predicted that the availability of piloting cues would result in lower errors for all locomotion interfaces, and especially so for discordant teleporting. However, the only piloting cues that facilitated performance were those that included a boundary, such as a fence or room 
walls. Collectively, these results indicate that geometric boundaries can improve spatial updating when using a teleporting interface and that landmarks can be helpful but only in the context of a geometric boundary.

The experiments that included outdoor landmarks indicate that landmarks are helpful primarily to provide orientation to a boundary. In Experiment 5, the circular fence alone was useless for determining self-orientation during travel due to its rotational symmetry. However, landmarks beyond the fence provided an orientation to the boundary that allowed for more accurate identification of self-orientation. We liken this finding to research on reference directions, whereby salient environmental features lead to selection of a reference direction that serves as a conceptual "north” (Kelly \& McNamara, 2008; Shelton \& McNamara, 2001), similar to the manner in which the "top" of an object is determined (Rock, 1973). Accordingly, landmarks provided an orientation to the boundary, even when the boundary itself was ambiguous. When moving through the environment, we speculate that participants updated their orientation in space relative to the reference direction defined by the boundary and landmarks.

We speculate that the environmental cues that facilitated performance compared to the open field (e.g., the classroom) were combined with cues available when traversing the outbound path. However, evidence for cue combination is limited by lack of a piloting-only condition in which participants return to a learned location in the presence of the environmental cues but without the benefit of traveling from the learned location to their current location. Research on cue combination typically includes single- and dual-cue conditions (Chen et al., 2017; Sjolund et al., 2018; Nardini et al., 2008), and cue combination is indicated by dual-cue performance that is better than performance in either single-cue condition. It is possible that participants using the discordant teleporting interface relied exclusively on piloting when available, but this strategy 
would be sub-optimal. Although discordant teleporting performance in the open field was not particularly accurate, it was better than chance $^{2}$, and therefore provided useful information that could be combined with piloting to improve accuracy. Partially concordant teleporting, compared to discordant teleporting, may have benefited less from cue combination because path information was more reliable due to the inclusion of body rotation and therefore received higher weight, leading to a correspondingly lower weight for piloting cues. There was no evidence that walking performance improved in the presence of environmental cues, although performance was numerically better with environmental cues compared to without. We speculate that the path cues from walking were much more reliable than the piloting cues, leading to relative weights that minimized the impact of piloting.

Given the importance of boundaries for accurate spatial updating, future research should investigate whether the form of the boundary affects its value as a cue to spatial updating. Architects have long considered that the design of spaces and subspaces can influence how people feel and behave, and the boundaries of those spaces can be physical (land, water, railroads), or simply “in people's minds" (Alexander et al., 1977). Boundary vector cells in the rodent brain respond to environmental boundaries such as walls or drop-offs (Lever et al., 2009). In humans, the representation of purely visual boundaries such as flooring transitions appears to be dissociable from navigational boundaries (Julian, Ryan, Hamilton, \& Epstein, 2016). It would be interesting to evaluate whether human spatial updating is primarily affected by navigationallyrelevant boundaries or whether visual boundaries are equally useful.

\footnotetext{
${ }^{2}$ It is difficult to specify chance performance using absolute distance error. However, chance performance in absolute angular error is clearly defined $\left(90^{\circ}\right)$. Combining data from Experiments 1 and 2 in the open field VE, analysis of absolute angular errors indicated that all locomotion interfaces produced performance better than chance $(p ’ s<.001)$, with performance ranging from $11.6^{\circ}\left(\mathrm{SE}=1.2^{\circ}\right)$ in the walking condition to $63.7^{\circ}\left(\mathrm{SE}=3.8^{\circ}\right)$ in the discordant teleporting condition.
} 
The success of virtual reality applications often depends on successful navigation through larger virtual environments, including ships, power plants, tourist destinations, and constructions sites. The teleporting interface is widely used in VR applications such as these, and its advantages include ease of use (Bozgeyikli et al., 2016; Langbehn et al., 2018) and low incidence of sickness Christou \& Aristidou, 2017; Moghadam et al., 2018; Langbehn et al., 2018; Weißker et al., 2018). However, the experiments reported here indicate that developers should be cautious about the potential for disorientation, especially when users teleport to change their location and orientation. When teleportation is used, environmental boundaries can mitigate but not eliminate the spatial cognitive consequences of teleporting, and landmarks can be helpful when presented in the context of boundaries. 


\section{References}

Alexander, C., Ishikawa, S., Silverstein, M., Jacobson, M., Fiksdahl-King, I., \& Angel, S. (1977). A Pattern Language: Towns, Buildings, Construction. New York: Oxford University Press.

Bowman, D. A., Koller, D., \& Hodges, L. F. (1997). Travel in immersive virtual environments: An evaluation of viewpoint motion control techniques. Proceedings of the 1997 Virtual Reality Annual International Symposium, 45-52.

Bozgeyikli, E., Raij, A., Katkoori, S., \& Dubey, R. (2016). Point \& teleport locomotion technique for virtual reality. Proceedings of the 2016 Annual Symposium on ComputerHuman Interaction in Play, 205-216. https://doi.org/10.1145/2967934.2968105

Burgess, N., Jackson, A., Hartley, T., \& O’Keefe, J. (2000). Predictions derived from modeling the hippocampal role in navigation. Biological Cybernetics, 83, 301-312.

Chance, S. S., Gaunet, F., Beall, A. C., \& Loomis, J. M. (1998). Locomotion mode affects the updating of objects encountered during travel: The contribution of vestibular and proprioceptive inputs to path integration. Presence: Teleoperators and Virtual Environments, 7, 168-178.

Chen, X., McNamara, T. P., Kelly, J. W., \& Wolbers, T. (2017). Cue combination in human spatial navigation. Cognitive Psychology, 95, 105-144. https://doi.org/10.1016/j.cogpsych.2017.04.003

Chrastil, E. R. \& Warren, W. H. (2012). Active and passive contributions to spatial learning. Psychonomic Bulletin \& Review, 19, 1-23.

Christou, C. G. \& Aristidou, P. (2017). Steering Versus Teleport Locomotion for Head Mounted Displays. In De Paolis, L., Bourdot, P., \& Mongelli, A. (Eds), Augmented Reality, Virtual 
Reality, and Computer Graphics: Lecture Notes in Computer Science, vol 10325 (pp. 431-446). Springer.

De Sousa, M. P. A., Filho, M. R., Nunes, M. V. A., \& Lopes, A. d. C. (2010). Maintenance and operation of a hydroelectric unit of energy in a power system using virtual reality. International Journal of Electrical Power \& Energy Systems, 32(6), 599-606.

Go, H., \& Gretzel, U. (2010). The role of interactive maps and spatial ability in creating virtual tourism experiences: a measurement framework. Paper presented at the Travel and Tourism Research Association (TTRA), San Antonio, TX.

Grant, S. C., \& Magee, L. E. (1998). Contributions of proprioception to navigation in virtual environments. Human Factors, 40(3), 489-497.

Grechkin, T., Thomas, J., Azmandian, M., Bolas, M. \& Suma, E. (2016). Revisiting detection thresholds for redirected walking: Combining translation and curvature gains. Proceedings of the ACM Symposium on Applied Perception, 113-120.

Hartley, T., Burgess, N., Lever, C., Cacucci, F., \& O’Keefe, J. (2000). Modeling place fields in terms of the cortical inputs to the hippocampus. Hippocampus, 10, 369-379.

Interrante, V., Ries, B., \& Anderson, L. (2007). Seven league boots: A new metaphor for augmented locomotion through moderately large scale immersive virtual environments. 3DUI '07: Proceedings of the 2007 IEEE Symposium on 3D User Interfaces, 167-170.

Julian, J. B., Ryan, J., Hamilton, R. H., \& Epstein, R. A. (2016). The occipital place area is causally involved in representing environmental boundaries during navigation. Current Biology, 26(8), 1104-1109. https://doi.org/10.1016/j.cub.2016.02.066.

Kearns, M. J., Warren, W. H., Duchon, A. P., \& Tarr, M. J. (2002). Path integration from optic flow and body senses in a homing task. Perception, 31, 349-374. DOI:10.1068/p3311 
Kelly, J.W. \& McNamara, T.P. (2008). Spatial memories of virtual environments: How egocentric experience, intrinsic structure, and extrinsic structure interact. Psychonomic Bulletin \& Review, 15(2), 322-327.

Kelly, J. W., McNamara, T. P., Bodenheimer, B., Carr, T. H., \& Rieser, J. J. (2008). The shape of human navigation: How environmental geometry is used in maintenance of spatial orientation. Cognition, 109(2), 281-286. doi:10.1016/j.cognition.2008.09.001

Klatzky, R. L., Loomis, J. M., Beall, A. C., Chance, S. S., \& Golledge, R. G. (1998). Spatial updating of self-position and orientation during real, imagined, and virtual locomotion. Psychological Science, 9, 293-298.

Langbehn, E., Lubos, P., \& Steinicke, F. (2018). Evaluation of locomotion techniques for roomscale VR: Joystick, teleportation, and redirected walking. Proceedings of the Virtual Reality International Conference 2018, 1-9. https://doi.org/10.1145/3234253.3234291.

Lever, C., Burton, S., Jeewajee, A., O’Keefe, J., \& Burgess, N. (2009). Boundary vector cells in the subiculum of the hippocampal formation. The Journal of Neuroscience, 29(31), 97719777. DOI:10.1523/JNEUROSCI.1319-09.2009

Mainelli, T., Shirer, M. \& Ubrani, J. (2019). Augmented reality and virtual reality headsets poised for significant growth. International Data Corporation. Retrieved from https://www.idc.com/getdoc.jsp?containerId=prUS44966319

Moghadam, K. R., Banigan, C., \& Ragan, E. D. (2018). Scene transitions and teleportation in virtual reality and the implications for spatial awareness and sickness. IEEE Transactions on Visualization and Computer Graphics https://doi.org/10.1109/TVCG.2018.2884468 
Moon, S., Becerik-Gerber, B., \& Soibelman, L. (2019). Virtual Learning for Workers in Robot Deployed Construction Sites. Advances in Informatics and Computing in Civil and Construction Engineering, 889-895.

Nardini, M., Jones, P., Bedford, R., \& Braddick, O. (2008). Development of cue integration in human navigation. Current Biology, 18(9), 689-693. https://doi.org/10.1016/j.cub.2008.04.021

O’Keefe, J., \& Burgess, N. (1996). Geometric determinants of the place fields of hippocampal neurons. Nature, 381, 425-428.

Padilla, L. M., Creem-Regehr, S. H., Stefanucci, J. K., \& Cashdan, E. A. (2017). Sex differences in virtual navigation influenced by scale and navigation experience. Psychonomic Bulletin \& Review, 24(2), 582-590. DOI 10.3758/s13423-016-1118-2

Riecke, B. E., van Veen, H. A. H. C., \& Bülthoff, H. H. (2002). Visual homing is possible without landmarks: A path integration study in virtual reality. Presence Teleoperators and Virtual Environments, 11(5), 443-473.

Rock, I. (1973). Orientation and form. New York: Academic Press.

Ruddle, R. A., \& Lessels, S. (2006). For efficient navigational search, humans require full physical movement, but not a rich visual scene. Psychological Science, 17(6), 460-465.

Ruddle, R. A., Volkova, E., \& Bülthoff, H. H. (2011). Walking improves your cognitive map in environments that are large-scale and large in extent. ACM Transactions on ComputerHuman Interaction, 18(2), 1-22. https://doi.org/10.1145/1970378.1970384

Ruddle, R. A., Volkova, E., Mohler, B., \& Bülthoff, H. H. (2011). The effect of landmark and body-based sensory information on route knowledge. Memory \& Cognition, 39(4), 686699. https://doi.org/10.3758/s13421-010-0054-Z 
Shelton, A. L., \& McNamara, T. P. (2001). Systems of spatial reference in human memory. Cognitive Psychology, 43, 274-310. doi:10.1006/cogp.2001.0758

Sjolund, L. A., Kelly, J. W., \& McNamara, T. P. (2018). Optimal combination of environmental cues and path integration during navigation. Memory \& Cognition, 46(1), 89-99. https://doi.org/10.3758/s13421-017-0747-7

Steinicke, F., Bruder, G., Jerald, J., Frenz, H., \& Lappe, M. (2010). Estimation of detection thresholds for redirected walking techniques. IEEE Transactions on Visualization and Computer Graphics, 16(1), 17-27.

Sun, H., Chan, G. S. W., \& Campos, J. L. (2004). Active navigation and orientation-free spatial representations. Memory \& Cognition, 32, 51-71.

Tate, D. L., Sibert, L., \& King, T. (1997). Virtual environments for shipboard firefighting training. Proceedings of IEEE 1997 Annual International Symposium on Virtual Reality, $61-68$

Valve Corporation (2016). The Lab.

Waller, D., Loomis, J. M., \& Haun, D. B. (2004). Body-based senses enhance knowledge of directions in large-scale environments. Psychonomic Bulletin \& Review, 11(1), 157-163.

Weißker, T., Kunert, A., Fröhlich, B., \& Kulik, A. (2018). Spatial updating and simulator sickness during steering and jumping in immersive virtual environments. 2018 IEEE Conference on Virtual Reality and 3d User Interfaces (VR), 97-104. https://doi.org/10.1109/VR.2018.8446620

Williams, B., Narasimham, G., McNamara, T. P., Carr, T. H., Rieser, J. J., \& Bodenheimer, B. (2006). Updating orientation in large virtual environments using scaled translational gain. 
Proceedings of the 3rd Symposium on Applied Perception in Graphics and Visualization, $21-28$.

Wraga, M., Creem-Regehr, S. H., \& Proffitt, D. R. (2004). Spatial updating of virtual displays during self- and display rotation. Memory \& Cognition, 32(3), 399-415.

Zhang, L. \& Mou, W. (2017). Piloting systems reset path integration systems during position estimation. Journal of Experimental Psychology: Learning, Memory, and Cognition, 43(3), 472-491. DOI: 10.1037/xlm0000324. 